\title{
Application of Dual-Energy Computed Tomography for Breast Cancer Diagnosis
}

\author{
Yukiko Okamura ${ }^{1,2}$, Nobuko Yoshizawa², Masaru Yamaguchi1, Ikuo Kashiwakura ${ }^{{ }^{*}}$ \\ ${ }^{1}$ Department of Radiological Life Sciences, Hirosaki University Graduate School of Health Sciences, Hirosaki, Japan \\ ${ }^{2}$ Department of Diagnostic Radiology and Nuclear Medicine, Hamamatsu University School of Medicine, Hamamatsu, Japan \\ Email: *ikashi@hirosaki-u.ac.jp
}

How to cite this paper: Okamura, Y., Yoshizawa, N., Yamaguchi, M. and Kashiwakura, I. (2016) Application of Dual-Energy Computed Tomography for Breast Cancer Diagnosis. International Journal of Medical Physics, Clinical Engineering and Radiation Oncology, 5, 288-297.

http://dx.doi.org/10.4236/ijmpcero.2016.54029

Received: September 17, 2016

Accepted: November 11, 2016

Published: November 14, 2016

Copyright $\odot 2016$ by authors and Scientific Research Publishing Inc. This work is licensed under the Creative Commons Attribution International License (CC BY 4.0).

http://creativecommons.org/licenses/by/4.0/

\begin{abstract}
The present study aimed to investigate the possibility of using dual-energy computed tomography (CT) before therapy to discriminate between normal breast tissue and tumor tissue in patients with breast cancer, without the need to use a contrast medium. The following patient data were extracted by interview and from the hospital's radiology information system: height, weight, age, menstrual cycle, CT images of normal tissue and tumors with or without contrast medium, and the histopathological diagnosis of the aspiration biopsy. The median age of the 43 participants was 56 years (range, 30 - 80 years). The CT values were evaluated using a clinical analytical program based on the three-material decomposition technique. Breast cancer was classified into ductal carcinoma in situ, invasive ductal carcinoma, invasive lobular carcinoma, fibromatosis-like metaplastic carcinoma, and apocrine carcinoma. In all conditions, regardless of contrast medium, the CT values of tumor tissues were higher than those of normal breast tissue, indicating the effectiveness of dual-energy CT (DE-CT) in the diagnosis of breast cancer. By contrast, DE-CT showed limited potential for distinguishing ductal carcinoma in situ from invasive ductal carcinoma. There have only been a few reports regarding CT examination of breast cancer, and it is expected this study encourage the development of DE-CT imaging to improve tumor detection in patients with breast cancer.
\end{abstract}

\section{Keywords}

Breast Cancer, Dual-Energy CT, CT Value, Contrast Medium, Oncological Imaging, Ductal Carcinoma in Situ, Invasive Ductal Carcinoma

\section{Introduction}

Breast cancer has traditionally been considered a Western disease, but in recent years 
its prevalence has been increasing annually among Japanese women as a consequence of changing lifestyles, notably with fewer marriages and more pregnancies at older ages. Currently, one-in-twelve Japanese women suffer from breast cancer in their lifetime. Breasts are composed of fatty and breast tissue that comprises ducts and lobules, with about $90 \%$ of breast cancers originating from the ducts. These ductal carcinomas are divided into either ductal carcinoma in situ (DCIS) or invasive ductal carcinoma (IDC) by the degree of invasion of cancer cells. Breast cancer is also typically responsive to available therapies, and complete cure can be expected when it is diagnosed at an early stage and managed with appropriate treatment. Therefore, breast cancer screening, such as mammography and ultrasound, is recommended once every two years to identify early-stage disease [1] [2].

Routine diagnostic imaging of the breast is typically by mammography, ultrasonography, or positron emission tomography-computed tomography (CT), with CT and magnetic resonance imaging (MRI) performed to obtain additional information or to support surgery [3] [4]. Regarding CT examination of breast tissue, there are only a few reports on the possibility of its use for qualitative diagnosis of breast cancer. By contrast, technical advances in MRI have improved MRI resolution and fat suppression techniques, so that the detection sensitivity of intraductal cancer infiltration is now high [3] [4] [5]; but, it is difficult to use these images for surgical support because of the shape of the receiver coil and the face-down position adopted. Compared with MRI, the advantages of CT imaging are that it is capable of imaging in a face-up (surgical) position, it has high spatial resolution, it has reduced inspection time (and burden on patients), and it can be used to search for distant metastasis (e.g., axillary lymph node, liver, lung, and bone) [5].

Recently, dual-energy CT (DE-CT) has also been developed [6], which uses two types of CT datasets acquired with different X-ray spectra generated by different tube potentials. This new technique is superior to standard CT based on 1) better image quality and tumor contrast; 2 ) the need for fewer images to obtain the same information as a single-energy study; 3 ) the associated reduction in the number of artifacts, such as beam hardening; and 4) the ability to discriminate between and quantify substances like iodine, calcium, and fat [7] [8]. In breast cancer, calcifications can be seen on a mammogram, and microcalcifications are an important type of calcification that reflect specks of calcium in areas of rapidly dividing cells. Therefore, because a clustering of microcalcifications may indicate cancer, DE-CT might better discriminate between normal breast tissue and tumor tissues and be useful for quantifying the amount of tumor tissue. Furthermore, although contrast medium has generally been considered essential for distinguishing normal breast tissue from tumor tissue, it is associated with allergy. DE-CT can obtain comparable image contrast to standard CT with a reduced need for contrast medium, thereby reducing this risk.

The present study aimed to investigate the possibility of using dual-energy computed tomography (CT) before therapy to discriminate between normal breast tissue and tumor tissue in patients with breast cancer, without the need to use a contrast medium. 
We investigated whether it was possible, before treatment to discriminate normal breast tissue from tumor tissue by DE-CT, without using contrast medium.

\section{Materials and Methods}

\subsection{Patients Characteristics}

This study was approved by the Committee of Medical Ethics of Hirosaki University Graduate School of Health Sciences (Hirosaki, Japan) and Hamamatu University Graduate School of Medicine (Hamamatu, Japan). Before all CT examinations, the study aim was explained to each patient and they were informed that there would be no negative consequences for either themselves or their family if they did not participate. A consent form was then signed by each participant.

Data were obtained for 83 patients with breast cancer who underwent CT examination at Hamamatsu University Hospital between July 2014 and March 2016. All of the data were summarized in Table 1 . Of these, 40 were excluded because of leakage of contrast medium, the image being out of the field of view (FOV) range, body motion, and masses with calcification. Therefore, 43 patients were included in the study, with a median age of 56 years (range, 30 - 80 years). The following data were extracted for each patient from interviews and from the hospital's radiology information system: height, weight, age, menstrual cycle, CT values of normal tissue and tumors (with or without contrast medium), and the histopathological diagnosis of the aspiration biopsy.

\subsection{CT Protocol}

X-ray CT examinations were performed using a SOMATOM Definition FLASH system (Siemens Medical Solutions, Forchheim, Germany) with the parameters shown in Table 1. Proprietaryiodine contrast, Iopamilon Injection Syringe (iopamidol, 300/370 mg

Table 1. CT examination protocol.

\begin{tabular}{cc}
\hline & Plain and contrast-enhanced DE-CT \\
\hline Tube voltage $(\mathrm{kV})$ & $100 / 140$ \\
Tube current & CT-auto exposure control \\
Quality ref. (mAs) & $250 / 193$ \\
Rotation time (sec) & 0.5 \\
Detector configuration (mm) & $128 \times 0.6(64 \times 0.6=38.4)$ \\
Pitch factor & 0.8 \\
Scan length (mm) & 450 \\
Recon kernel & D30f \\
Slice $(\mathrm{mm})$ & 5 \\
Increment $(\mathrm{mm})$ & 5 \\
Acquisition time (sec) & 16 \\
Field of view $(\mathrm{mm})$ & 337 \\
\hline
\end{tabular}


iodine/ml, Bayer, Leverkusen, German) or the OMNIPAQUE Injection Syringe (iohexol, $300 \mathrm{mg}$ iodine/ml, Daiichi-Sankyo Co, Ltd., Tokyo, Japan), was injected using a Dual Shot GX 7 (Nemoto, Japan) in $540 \mathrm{mg}$ iodine/ml over 50 seconds, before the CT scan was initiated 80 seconds after administration.

\subsection{Evaluation of CT Values}

All CT values were evaluated using a Siemens Medical Solutions analytical program based on the three-materials decomposition technique. The CT data were collected from the hospital medical information system with and without contrast medium at two tube voltages $(100 \mathrm{kV}$ and $140 \mathrm{kV})$. Data with or without contrast medium were derived from 43 normal breast tissues and from 43 patients with cancer. The region of interest was set at $4 \mathrm{~mm}$ in diameter per image. Further, the CT values for $120 \mathrm{kV}$ were derived from the combined images of the $100 \mathrm{kV}$ and $140 \mathrm{kV}$ tube voltages.

\subsection{Histopathological Classification of Breast Cancer}

As shown in Table 2, 43 patients were classified into five groups based on a previous report [9]: DCIS, IDC, invasive lobular carcinoma, fibromatosis-like metaplastic carcinoma, and apocrine carcinoma.

\subsection{Statistical Analysis}

Statistical significance was estimated using the Mann-Whitney $U$-test or Student's $t$-test. Significances levels were calculated by Microsoft Excel 2013 (Microsoft, Redmond, WA, USA) with the StatFlex version 6.0 add-in (Artech Co., Ltd., Osaka, Japan). A value of $P<0.05$ was considered statistically significant.

\section{Results}

\subsection{Comparison of CT Values for Normal Breast Tissue and Tumor Tissue}

The CT values for normal breast tissue and tumor tissue at the three different tube voltages $(100 \mathrm{kV}, 120 \mathrm{kV}$, and $140 \mathrm{kV})$, with or without contrast medium, are summarized

Table 2. Histopathological diagnoses.

\begin{tabular}{ccc}
\hline & Histopathology diagnosis & Number of patients \\
\hline \multirow{2}{*}{ Malignant } & IDC & 28 \\
& ILC & 5 \\
Decif & Fibromatosis-like & 6 \\
& metaplastic carcinoma & 2 \\
& Apocrine carcinoma & 2 \\
& Total & 43 \\
\hline
\end{tabular}

IDC: invasive ductal carcinoma, DCIS: ductal carcinoma in situ, ILC: invasive lobular carcinoma. 
in Table 3 and Figure 1. The CT values with contrast medium were significantly higher than those without contrast medium in most conditions, regardless of tissue type. The CT values obtained in normal breast tissue with contrast medium showed an approximate 1.1-fold increase in the tube voltage compared with those obtained without contrast medium. In the case of tumors, the CT value with contrast medium showed a 1.7to 2.4-fold increase, indicating the effectiveness of contrast medium. The fold-increase decreased with each increase in tube voltage, such that there was a 2.36-fold increase for $100 \mathrm{kV}$, a 2.05 -fold increase for $120 \mathrm{kV}$, and a 1.72 -fold increase for $140 \mathrm{kV}$.

Furthermore, the CT values for normal breast tissue were significantly lower than those for tumor tissue under all conditions, regardless of contrast medium use, indicating the effectiveness of DE-CT for breast cancer diagnosis. In the case of nonadministration of contrast medium, the normal-to-tumor CT ratio was approximately 1.5-times for all tube voltages. When contrast medium was administered, the normalto-tumor ratio for CT values increased to 2.3- to 3.0-fold, showing that the CT value of tumor tissue was higher than that of normal tissue; the fold-increase again decreased with increasing tube voltages.

Table 3. CT values, with and without contrast medium, for normal breast tissue and tumor tissue.

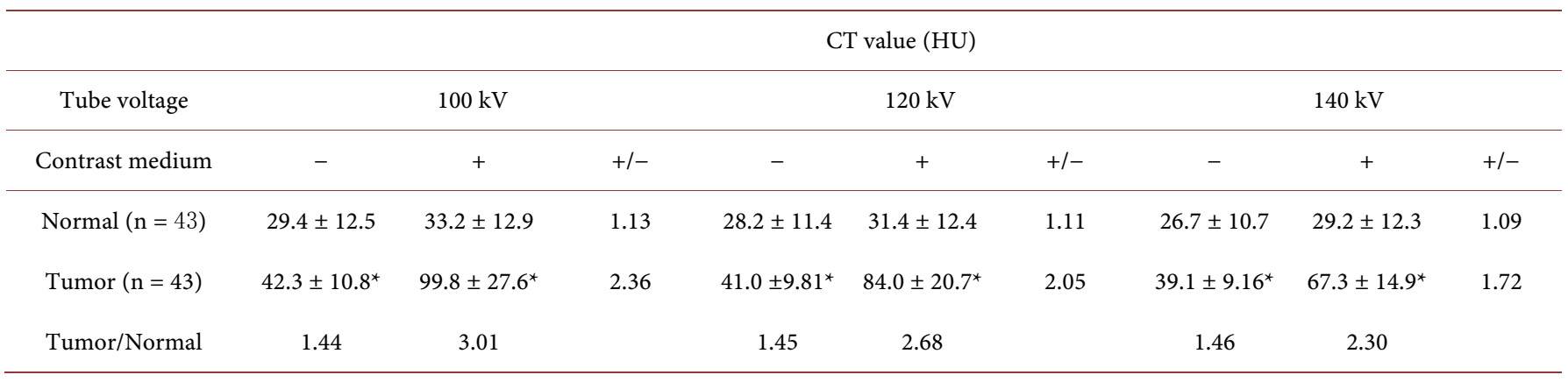

Each CT value (Hounsfield unit; HU) was obtained by three different voltage conditions, as shown in the table. The data are expressed as the means \pm SD. The values shown in parentheses are the number of patients. The value shown in parentheses is the number of patients. ${ }^{*} \mathrm{P}<0.05$ by Mann-Whitney's $U$-test in the comparison with normal mammalian gland $v s$ tumor.

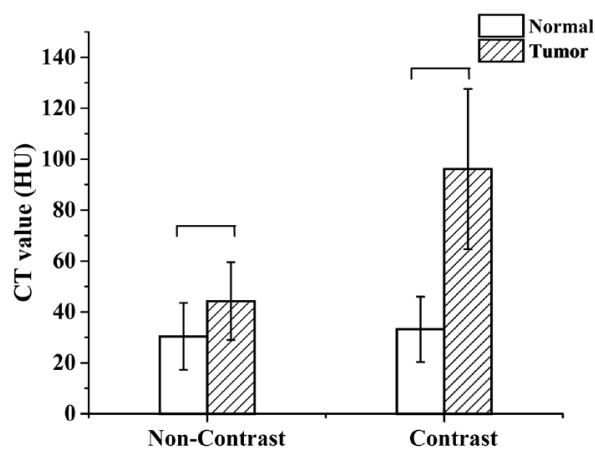

(a)

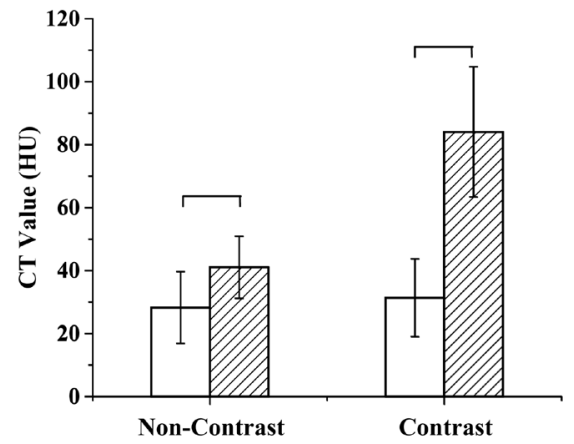

(b)

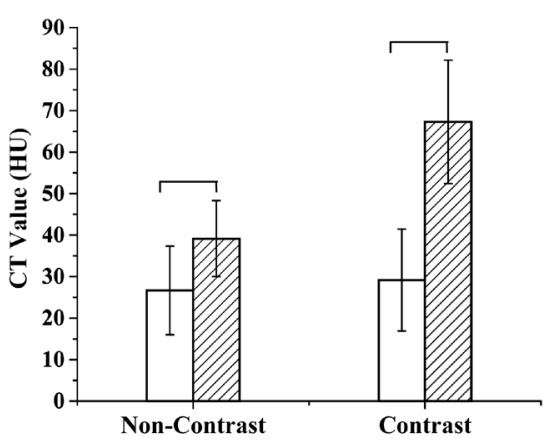

(c)

Figure 1. Evaluation of CT values in the normal mammary gland and tumors $(\mathrm{n}=43)$. The CT value of $100 \mathrm{Kv}(\mathrm{a})$; The CT value of 120 $\mathrm{Kv}$ (b); The CT value of $140 \mathrm{Kv}$ (c). The CT value was analyzed by VNC. Data are expressed as mean \pm S.D. ${ }^{\star} \mathrm{P}<0.05$ by Mann-Whitney's $U$-test. 


\subsection{Comparison of CT Values between Patients with DCIS and IDC}

The CT values of patients with DCIS and IDC obtained under the three different conditions, and with or without contrast medium, are summarized in Table 4 and Figure 2. As with the comparison of normal and tumorous breast tissue, contrast medium enhanced the CT values in all conditions, regardless of whether DCIS or IDC was diagnosed. When the CT values of patients with DCIS and IDC were compared, there were no statistically significant differences in most areas; however, for the values with contrast medium injection, DE-CT showed no effectiveness in either condition. The CT values obtained with contrast medium were shown to decrease as the tube voltage increased, moving from a 2.55 -fold increase to a 1.86 -fold increase. Thus, the foldincrease for CT with and without contrast medium also decreased as the tube voltage increased.

\section{Discussion}

In the present study, the possibility of using DE-CT before therapy to discriminate between normal breast tissue and tumor tissue, without the aid of contrast medium, was investigated. It was shown that contrast medium significantly enhanced all CT values, as shown in Table 3 and Table 4, demonstrating the effectiveness of the contrast medium. However, regardless of contrast medium use, it was also shown that the CT val-

Table 4. CT values, with and without contrast medium, for patients with DCIS and IDC.

\begin{tabular}{|c|c|c|c|c|c|c|c|c|c|}
\hline \multirow{2}{*}{$\begin{array}{c}\text { Tube voltage } \\
\text { Contrast medium }\end{array}$} & \multicolumn{9}{|c|}{$\mathrm{CT}$ value (HU) } \\
\hline & - & + & $+/-$ & - & + & $+1-$ & - & + & $+1-$ \\
\hline DCIS $(\mathrm{n}=6)$ & $38.9 \pm 10.8$ & $99.2 \pm 22.3$ & 2.55 & $37.7 \pm 9.9$ & $82.8 \pm 15.2$ & 2.18 & $35.6 \pm 9.36$ & $66.1 \pm 9.60$ & 1.86 \\
\hline IDC $(\mathrm{n}=28)$ & $43.1 \pm 11.7$ & $102.9 \pm 28.6$ & 2.39 & $41.6 \pm 10.5$ & $86.2 \pm 21.9$ & 2.07 & $39.6 \pm 9.70$ & $69.1 \pm 15.8$ & 1.74 \\
\hline
\end{tabular}

Each CT value (Hounsfield unit; HU) was obtained by three different voltage conditions, as shown in the table. The data are expressed as the means \pm SD. The value shown in parentheses are the number of patients.

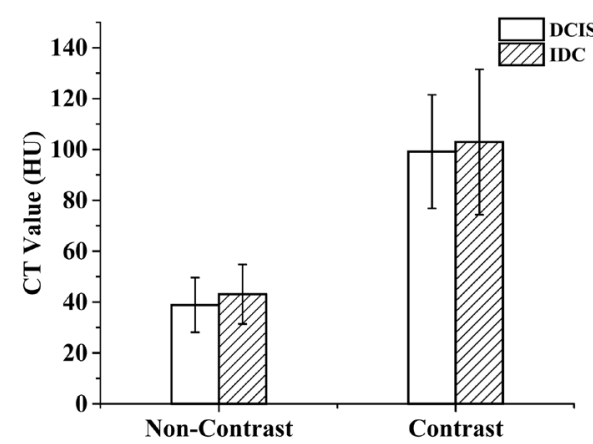

(a)

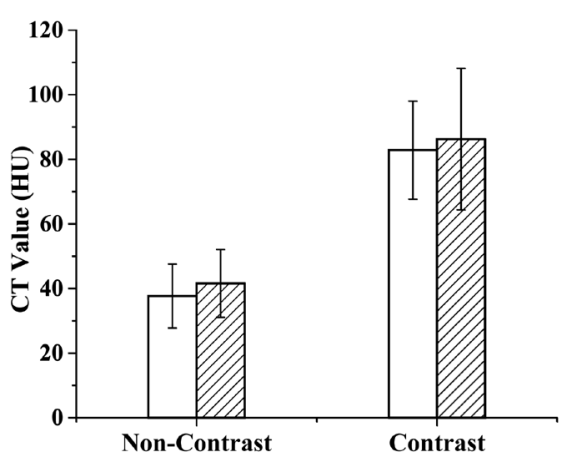

(b)

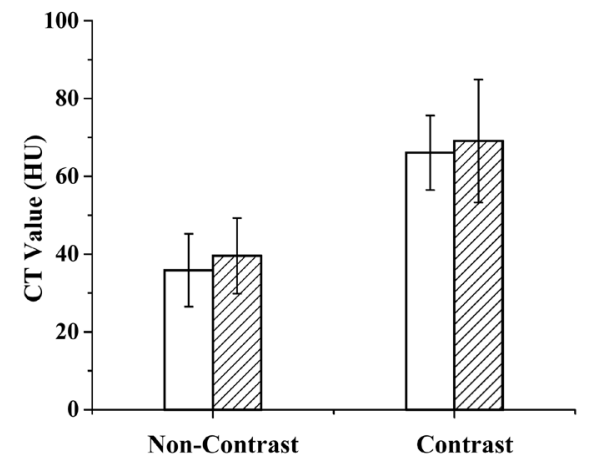

(c)

Figure 2. Evaluation of CT values in the DCIS $(n=6)$ and IDC $(n=29)$ in non-contrast enhanced and contrast enhanced. The CT value of $100 \mathrm{Kv}(\mathrm{a})$; The CT value of $120 \mathrm{Kv}$ (b); The CT value of $140 \mathrm{Kv}$ (c). The CT value was analyzed by VNC. Data are expressed as mean \pm S.D. 
ues of tumor tissues were still higher than those of normal breast tissue (Table 3, Figure 1), which indicated the effectiveness of DE-CT for diagnosing breast cancer.

Breast CT examinations may be useful for diagnosing the spread of breast cancer and determining the extent of surgical resection necessary. However, such examination places the patient at risk of radiation exposure and contrast medium allergy. As Tsushima et al. described, CT is the greatest source of medical radiation exposure in the general population, and is a potentially significant source of increased cancer risk [10]. They pointed out that CT examinations of the chest and upper abdomen contributed to approximately $73.2 \%$ of the collective dose from all CT examinations. The annual effective dose per capita in Japan has been estimated at 2.20 millisievert (mSv). In addition, Agrawal et al. pointed out the need to minimize the risks of contrast medium exposure in oncology patients [11], showing that the amount of contrast medium administered for CT examination, for either initial or follow-up evaluation, is a particular problem for those with compromised renal function [12] [13] [14]. In patients with cancer, advanced age and cumulative risk factors increase the susceptibility to adverse contrast material-related renal events; risk factors include, diabetes, hypertension, hyperlipidemia, and a history of nephrectomy. Therefore, lowering the iodine dose or the volume of a contrast material, without compromising the diagnostic quality of abdominal CT images, is desirable [11]. From this perspective, as Simons et al. reported, DE-CT may have considerable potential for improving tumor detection and lesion characterization, while concurrently shortening the scan time, reducing the radiation dose, and decreasing exposure to the contrast medium [15].

DCIS is the most common type of non-invasive breast cancer. It is a heterogeneous, unicentric precursor of invasive breast cancer that is frequently identified through core needle biopsy and mammography in breast screening programs [16]. By contrast, IDC of the breast comprises a group of malignant epithelial tumors that are characterized by invasion into adjacent tissues and a tendency to metastasize to distant sites, including lymph nodes [17]. The progression from proliferative disease without atypia to atypical hyperplasia, from atypical hyperplasia to DCIS, and from DCIS to IDC has been proposed by a number of investigators to be a possible model for the development of invasive ductal carcinoma in humans, despite continued controversies [18]. Regardless, the present study showed that DE-CT has limited potential for differentiating DCIS and IDC (Table 4, Figure 2). Although the present study evaluated the potential of DE-CT for diagnosing breast cancer by CT alone, it will be necessary to verify the findings by comparing CT values and images.

The incidence and mortality rate for breast cancer in Japan has been increasing since the middle of the 1980s [19]. In 2014, it was the fifth leading cause of cancer mortality, with 13,240 deaths, accounting for 9\% of all cancer deaths in women; and in 2015, it was the most common cancer in Japanese women, with the estimated 89,400 cases representing $21 \%$ of all female cancers. [20]. The age-standardized incidence rate peaks at 45 - 49 years old, which is much younger than in Western women [11]. Routine mammography and breast examination has been recommended in Japan since 2000 
[21]. However, there are some problems with breast examination, including its precision. Difficulties in lesion detection have been reported during mammography, particularly on account of high-density mammary gland tissue (dense breast) which is commonly seen in younger age groups [22]. Several studies have examined automated segmentation of mammary gland regions by torso CT images [23] and differentiation of malignant and benign incidental breast lesion detected by Multidetector-Row CT [24], although there was no analysis of the CT value in mammary gland and tumors by using DE-CT.

The dual-source, DE-CT scanners used in the present study consist of two X-ray tubes that are orthogonally mounted in one gantry and two sets of corresponding detectors that simultaneously acquire and process data with different energies (low and high) [11]. This scanner uses a three-material (soft tissue, fat, and contrast medium) decomposition algorithm. Ma et al. previously demonstrated that the separation of hepatic iron from fat, using dual-source, DE-CT material decomposition with a threematerial algorithm, was feasible in vivo, even when iron and fat coexisted [25]. Currently, DE-CT applications in clinical settings are mainly used in chest, abdomen, and genitourinary diagnosis, and have been used since the DE-CT technique first became available on commercial CT machines [11]. Very recently, Uhrig et al. reported that abdominal DE-CT was feasible without increasing radiation exposure or deteriorating the image quality, even when compared to dose-optimized single-energy CT (e.g., automatic voltage control). They concluded that DE-CT could contribute to sophisticated oncological imaging without a dose penalty [26].

Future application of DE-CT is anticipated in other specialties, although there is still a lack of comprehensive evidence for its use in the brain, head and neck, musculoskeletal tissue, and skin tissue. However, the potential of DE-CT in the context of oncological imaging has clearly not been fully exploited. In this study, it was shown that the CT values of tumor tissues were still higher than those of normal breast tissue regardless of contrast medium use, which indicated the effectiveness of DE-CT for diagnosing and discovering the breast cancer without any contrast mediums. It took a considerable amount of time to practical use due to technical constraints of DE-CT, but that will be introduced into a lot of facilities in the future. Breast CT imaging with DE-CT is expected to improve tumor detection in the future, but additional approaches will be required to resolve existing problems.

\section{Acknowledgements}

The authors thank the staff of the department of Diagnostic Radiology and Nuclear Medicine Hamamatsu University School of Medicine and Hospital for their support in collecting clinical data.

\section{References}

[1] Ohuchi, N., Suzuki, A., Sobue, T., Kawai, M., Yamamoto, S., Zheng, Y.F., Shiono, Y.N., Saito, H., Kuriyama, S., Tohno, E., Endo, T., Fukao, A., Tsuji, I., Yamaguchi, T., Ohashi, Y., 
Fukuda, M., Ishida, T. and J-START Investigator Groups (2016) Sensitivity and Specificity of Mammography and Adjunctive Ultrasonography to Screen for Breast Cancer in the Japan Strategic Anti-Cancer Randomized Trial (J-START): A Randomised Controlled Trial. Lancet, 387, 341-348. http://dx.doi.org/10.1016/S0140-6736(15)00774-6

[2] Kopans, D.B. and Swann, C.A. (1989) Preoperative Imaging-Guided Needle Placement and Localization of Clinically Occult Breast Lesions. $A J R, 152,1-9$.

http://dx.doi.org/10.2214/ajr.152.1.1

[3] Simauchi, A., Yamada, T., Sato, A., Takase, K., Usami, S., Ishida, T., Moriya, T. and Takahashi, S. (2006) Comparison of MDCT and MRI for Evaluating the Intraductal Component of Breast Cancer. American Journal of Roentgenology, 187, 322-329.

http://dx.doi.org/10.2214/AJR.05.0876

[4] Brown, J.C., Kontos, D., Schnall, M.D., Wu, S. and Schmitz, K.H. (2016) The Dose-Response Effects of Aerobic Exercise on Body Composition and Breast Tissue among Women at High Risk for Breast Cancer: A Randomized Trial. Cancer Prevention Research (Phila), 9, 581-588. http://dx.doi.org/10.1158/1940-6207.CAPR-15-0408

[5] Chang, C.H., Sibala, J.L., Fritz, S.L., Dwyer 3rd., S.J., Templeton, A.W., Lin, F. and Jewell, W.R. (1980) Computed Tomography in Detection and Diagnosis of Breast Cancer. Cancer, 46, 39-46.

http://dx.doi.org/10.1002/1097-0142(19800815)46:4+<939::AID-CNCR2820461315>3.0.CO i2-L

[6] Johnson, T.R. (2012) Dual-Energy CT: General Principles. American Journal of Roentgenology, 199, S3-S8. http://dx.doi.org/10.2214/AJR.12.9116

[7] Morgan, D.E. (2014) Dual-Energy CT of the Abdomen. Abdominal Imaging, 39, 108-134. http://dx.doi.org/10.1007/s00261-013-0033-5

[8] Miyanishi, S. (2010) Dual Energy Imaging of Advantage by Dual Source CT. http://www.innervision.co.jp/suite/siemens/technote/100704/index.html

[9] National Breast Cancer Foundation, Inc. (2016) Breast Cancer Types. http://www.nationalbreastcancer.org/types-of-breast-cancer

[10] Tsushima, Y., Taketomi-Takahashi, A., Takei, H., Otake, H. and Endo, K. (2010) Radiation Exposure from CT Examinations in Japan. BMC Medical Imaging, 10, 24.

http://dx.doi.org/10.1186/1471-2342-10-24

[11] Agrawal, M.D., Pinho, D.F., Kulkarni, N.M., Hahn, P.F., Guimaraes, A.R. and Sahani, D.V. (2014) Oncologic Applications of Dual-Energy CT in the Abdomen. Radiographics, 34, 589-612. http://dx.doi.org/10.1148/rg.343135041

[12] Thomsen, H.S. and Morcos, S.K. (2003) Contrast Media and the Kidney: European Society of Urogenital Radiology (ESUR) Guidelines. The British Journal of Radiology, 76, 513-518. http://dx.doi.org/10.1259/bjr/26964464

[13] Gleeson, T.G. and Bulugahapitiya, S. (2004) Contrast-Induced Nephropathy. American Journal of Roentgenology, 183, 1673-1689. http://dx.doi.org/10.2214/ajr.183.6.01831673

[14] Ng, C.S., Shaw, A.D., Bell, C.S. and Samuels, J.A. (2010) Effect of IV Contrast Medium on Renal Function in Oncologic Patients Undergoing CT in ICU. American Journal of Roentgenology, 195, 414-422. http://dx.doi.org/10.2214/AJR.09.4150

[15] Simons, D., Kachelriess, M. and Schlemmer, H.P. (2014) Recent Developments of DualEnergy CT in Oncology. European Radiology, 24, 930-939.

http://dx.doi.org/10.1007/s00330-013-3087-4

[16] Espina, V., Mariani, B.D., Gallagher, R.I., Tran, K., Banks, S., Wiedemann, J., Huryk, H., Mueller, C., Adamo, L., Deng, J., Petricoin, E.F., Pastore, L., Zaman, S., Menezes, G., Mize, 
J., Johal, J., Edmiston, K. and Liotta, L.A. (2010) Malignant Precursor Cells Pre-Exist in Human Breast DCIS and Require Autophagy for Survival. PLoS One, 5, e10240. http://dx.doi.org/10.1371/journal.pone.0010240

[17] Miki, Y., Suzuki, T. and Sasano, H. (2009) Intracrinology of Sex Steroids in Ductal Carcinoma In Situ (DCIS) of Human Breast: Comparison to Invasive Ductal Carcinoma (IDC) and Non-Neoplastic Breast. The Journal of Steroid Biochemistry and Molecular Biology, 114, 68-71. http://dx.doi.org/10.1016/j.jsbmb.2008.12.021

[18] London, S.J., Connolly, J.L., Schnitt, S.J. and Colditz, G.A. (1992) A Prospective Study of Benign Breast Disease and the Risk of Breast Cancer. JAMA, 267, 941-944. http://dx.doi.org/10.1001/jama.1992.03480070057030

[19] Sauvaget, C., Nishino, Y., Konno, R., Tase, T., Morimoto, T. and Hisamichi, S. (2016) Challenges in Breast and Cervical Cancer Control in Japan. The Lancet Oncology, 17, e305e312. http://dx.doi.org/10.1016/s1470-2045(16)30121-8

[20] National Cancer Centre (2016) Cancer Registry Data Online. http://ganjoho.jp/reg stat/index.html

[21] Hamashima, C., Ohta, K., Kasahara, Y., Katayama, T., Nakayama, T., Honjo, S. and Ohnuki, K. (2015) A Meta-Analysis of Mammographic Screening with and without Clinical Breast Examination. Cancer Science, 106, 812-818. http://dx.doi.org/10.1111/cas.12693

[22] Kuroki-Suzuki, S., Kuroki, Y., Ishikawa, T., Takeo, H. and Moriyama, N. (2010) Diagnosis of Breast Cancer with Multidetector Computed Tomography: Analysis of Optimal Delay Time after Contrast Media Injection. Clinical Imaging, 34, 14-19.

http://dx.doi.org/10.1016/j.clinimag.2009.03.004

[23] Kakkad, S.M., Solaiyappan, M., Argani, P., Sukumar, S., Jacobs, L.K., Leibfritz, D., Bhujwalla, Z.M. and Glunde, K. (2012) Collagen I Fiber Density Increases in Lymph Node Positive Breast Cancers: Pilot Study. Journal of Biomedical Optics, 17, Article ID: 116017. http://dx.doi.org/10.1117/1.jbo.17.11.116017

[24] Lin, Y.P., Hsu, H.H., Ko, K.H., Chu, C.M., Chou, Y.C., Chang, W.C. and Chang, T.H. (2016) Differentiation of Malignant and Benign Incidental Breast Lesions Detected by Chest Multidetector-Row Computed Tomography: Added Value of Quantitative Enhancement Analysis. PLOS ONE, 11, e0154569. http://dx.doi.org/10.1371/journal.pone.0154569

[25] Ma, J., Song, Z.Q. and Yan, F.H. (2014) Separation of Hepatic Iron and Fat by Dual-Source Dual-Energy Computed Tomography Based on Material Decomposition: An Animal Study. PLoS ONE, 9, e110964. http://dx.doi.org/10.1371/journal.pone.0110964

[26] Uhrig, M., Simons, D., Kachelrieß, M., Pisana, F., Kuchenbecker, S. and Schlemmer, H.P. (2016) Advanced Abdominal Imaging with Dual Energy CT Is Feasible without Increasing Radiation Dose. Cancer Imaging, 16, 15. http://dx.doi.org/10.1186/s40644-016-0073-5 
Submit or recommend next manuscript to SCIRP and we will provide best service for you:

Accepting pre-submission inquiries through Email, Facebook, LinkedIn, Twitter, etc.

A wide selection of journals (inclusive of 9 subjects, more than 200 journals)

Providing 24-hour high-quality service

User-friendly online submission system

Fair and swift peer-review system

Efficient typesetting and proofreading procedure

Display of the result of downloads and visits, as well as the number of cited articles

Maximum dissemination of your research work

Submit your manuscript at: http://papersubmission.scirp.org/

Or contact ijmpcero@scirp.org 